\title{
Dynamics of multiphoton excitation and quantum diffusion in Rydberg atoms
}

\author{
Kwanghsi Wang and Shih-I Chu \\ Department of Chemistry, University of Kansas, Lawrence, Kansas 66045
}

(Received 3 June 1988)

\begin{abstract}
We present a detailed two-dimensional (2D) quantal study of the dynamical evolution of microwave-driven Rydberg $\mathrm{H}$ atoms. We examine the range of validity of the conventional onedimensional (1D) models and explore the frequency- and intensity-dependent excitation and ionization mechanisms. The main findings of this paper can be summarized as follows: (i) The excitation spectra of Rydberg $\mathbf{H}$ atoms are strongly frequency dependent and can be roughly grouped into three characteristically different regions, each with a different excitation mechanism. In this paper, we emphasize the study of the two major excitation mechanisms: quantum diffusion and multiphoton resonant excitation. The region dominated by quantum diffusion lies in the frequency range $\omega_{c}<\omega_{0}<\omega_{d}$, where $\omega_{0}$ is the rescaled field frequency $\left(\omega_{0}=\omega n_{0}^{3} ; n_{0}\right.$ is the principal quantum number of the initial state); $\omega_{c}$, the classical chaotic threshold; and $\omega_{d}$, the quantum delocalization border. In this region, quasienergy levels are strongly perturbed and mixed and excitation is efficient, leading to the so-called underthreshold photoelectric ionization phenomenon. On the other hand, we found a series of frequency regions (in $\omega_{0}>\omega_{d}$ ) where the ionization is mainly due to multiphoton resonant excitation through the more isolated quasienergy avoided crossing points. (ii) The excitation pathways (1D versus 2D) are strongly intensity dependent. For microwave (rescaled) field strength $\varepsilon_{0}\left(\equiv \varepsilon n_{0}^{4}\right)$ in the range $\varepsilon_{c}<\varepsilon_{0}<\varepsilon_{q}$ (where $\varepsilon_{c}$ is the onset of classical chaos and $\varepsilon_{q}$ the quantum delocalization threshold), large discrepancies exist between $1 \mathrm{D}$ and $2 \mathrm{D}$ results. It is found that the 1D model seriously underestimates the ionization probabilities and, more importantly, the dominant channels for Rydberg atom excitation and ionization proceed through $n_{2}>0$ ladders rather than the $n_{2}=0$ ladder, as often assumed in the 1D model. As field strength increases above $\varepsilon_{q}$, however, the 1D model improves significantly. (iii) The quantum localization phenomenon is observed in the classically chaotic region $\left(\omega_{c}<\omega_{0}<\omega_{d}\right)$ when the field strength $\varepsilon_{0}$ is less than $\varepsilon_{q}$. However, quantum delocalization can appear when $\varepsilon_{0}>\varepsilon_{q}$. (iv) The stability of quantum diffusive motion is analyzed in terms of the quantal phase-space diagram and the autocorrelation function. The results lend support to the view that quantum mechanics can impose limitations on classical chaotic motion. (v) The way of turning on the field $(\sin \omega t$ or $\cos \omega t)$ does not affect significantly the dynamical evolution of the system. (vi) Finally, a computationally powerful new technique, invoking the use of artificial intelligence algorithms as well as the generalized Van Vleck perturbation theory for effectively reducing the dimensionality of the Floquet matrix, is introduced to facilitate the study of multiphoton resonant excitation of Rydberg atoms.
\end{abstract}

\section{INTRODUCTION}

The study of the dynamical evolution of highly excited hydrogen atoms driven by strong microwave fields is currently a subject of considerable interest both experimentally $^{1}$ and theoretically. ${ }^{2-9}$ Because of the complexity of the problem, one-dimensional (1D) models ${ }^{2,4-9}$ were often introduced to simplify the dynamics. Use of the classical dynamics shows that the system undergoes a transition from regular to chaotic motions ${ }^{2,4,6}$ as the field strength exceeds a critical value $\varepsilon_{c}$, leading to diffusive ionization of the atom. On the other hand, quantal studies of the same 1D Hamiltonian show that quantum interference can lead to significant inhibition of the diffusive motion in energy space, ${ }^{7}$ and that the quantual motion is stable ${ }^{8}$ even though it can be diffusive. Recent$\mathrm{ly}$, it has been further found that large-amplitude ionization can occur at frequencies much below those required for the one-photon photoelectric effect. ${ }^{9}$

To examine the scope of the validity of the 1D models and to explore the detailed frequency- and intensity- dependent excitation and ionization mechanisms, we have performed a detailed two-dimensional (2D) ab initio quantum-mechanical study of the nonlinear dynamical evolution of the microwave-driven Rydberg states of hydrogen atoms near the onset of classical chaos. The 2D results show significant departures from the strict 1D model in some intensity regimes regarding the time development of Rydberg states as well as the most probable ionization pathways. Further, it is found that ionization mechanisms are strongly frequency dependent. There are generally two different mechanisms operating in the excitation of Rydberg atoms: multiphoton resonant excitation and quantum diffusion. The former occurs when the separation of two nonadjacent quantum states (say, $|i\rangle$ and $|j\rangle)\left|E_{i}-E_{j}\right| \cong N \hbar \omega$ and simultaneous absorption (or emission) of $N$ photons can take place. On the other hand, quantum diffusion arises from a series of successive single-photon absorption and/or emission between adjacent levels. The physical origin of these phenomena can be understood by the examination of their quasi-energylevel diagrams. 
In the next section (Sec. II), we shall define the problem and the Hamiltonian, and outline the numerical methods for obtaining the solutions. A detailed investigation of the frequency- and intensity-dependent excitation dynamics of Rydberg $\mathrm{H}$ atoms is presented along with the examination of the validity of the $1 \mathrm{D}$ model in Sec. III. The quantal phase-space diagram and autocorrelation function are introduced to analyze the nature of quantum diffusive motion in the classically chaotic region. Finally in Sec. IV, we present a new numerical technique to facilitate the study of multiphoton resonant excitation dynamics.

These studies were greatly facilitated by access to the CRAY-2 supercomputer and by the recent advancements in Floquet methods. ${ }^{10,11}$ Some of the preliminary results have been reported previously. ${ }^{12}$

\section{THEORY}

The dynamical evolution of the hydrogen atom driven by an external linearly polarized monochromatic electric field is governed by the time-dependent Schrödinger equation (in atomic units)

$$
i \partial_{t} \psi(t)=\hat{H}(t) \psi(t)
$$

where $\hat{H}(t)$ is the periodic Hamiltonian given by

$$
\hat{H}(t)=-\frac{1}{2} \nabla^{2}-1 / r-\varepsilon(t) z,
$$

where $\varepsilon(t)=\varepsilon \sin \omega t$ or $\varepsilon \cos \omega t$, and $\varepsilon$ and $\omega$ are the microwave electric field strength (assuming $\varepsilon \| \hat{\mathbf{z}}$ ) and frequency, respectively. The quantum dynamics can be expediently studied in parabolic coordinates as the unperturbed solution is separable in these coordinates. Following previous theoretical studies, ${ }^{2,6-9}$ we shall assume that the hydrogen atom is initially prepared in a very elongated quantum state having parabolic quantum number at $n_{1}=n_{0}-1, n_{2}=0, m=0$, where $n_{0}(>>1)$ is the principal quantum number $n$ of the initial state. Since the magnetic quantum number $m$ is a constant of motion ( $m=0$ in this case), and since $n=n_{1}+n_{2}+|m|+1$, only two quantum numbers $\left(n_{1}\right.$ and $n_{2}$ or $n$ and $n_{2}$ ) can be varied during the excitation. Thus the quantum system under study is essentially a two-dimensional one.

In Fig. 1, we depict the energy-level diagram and the initial population flow direction for a hydrogen atom initially prepared in the $\left(n=66, n_{2}=0\right)$ quantum state. Not all the allowed transitions are shown. The heavy vertical arrows indicate the flowing paths in the conventional one-dimensional model ${ }^{2,4-9}$ (i.e., only the $n_{2}=0$ ladder is considered). The complete solution of the problem involves $2 \mathrm{D}$ dynamics and population is allowed to flow into $n_{2} \neq 0$ ladders as well. Owing to the dipole selection rule, horizontal (i.e., $\Delta n=0, \Delta n_{2} \neq 0$ ) transitions are not allowed, and Fig. 1 shows a forbidden zone in the initial short-time regime. However, once the population leaks into the $n_{2} \neq 0$ ladders [for example, via the dotted arrow (i.e., $\Delta n \pm 1, \Delta n_{2}= \pm 1$ ) transitions], rapid vertical flows within an $n_{2}$ ladder will fill up the forbidden zone. Alternatively, the forbidden zone can be filled up by first making the vertical transitions $\left(\Delta n= \pm 1, \Delta n_{2}=0\right)$ followed by the $\Delta n=\mp 1,\left|\Delta n_{2}\right|=1$ transitions (arrows not shown). The existence of the forbidden zone lasts typically about 0.1 ps. In general, the vertical couplings $\left(\Delta n= \pm 1, \Delta n_{2}=0\right)$ are one to two orders of magnitude larger than the dotted couplings $\left(\Delta n= \pm 1, \Delta n_{2}= \pm 1\right)$. This is the basis of justification for the conventional 1D models. However, since the microwave-driven Rydbergatom excitation is a dynamical process, a detailed 2D study is significant to the understanding of the genuine excitation pathways and ionization mechanisms.

The 2D quantum solution of the electronic motion, Eq. (1), has been solved by two different methods. In the first method, we expand $\psi(t)$ in terms of unperturbed hydrogenic eigenstates. This yields the following set of coupled channel equations:

$$
i \dot{C}_{n_{1} n_{2}}(t)=E_{n_{1} n_{2}} C_{n_{1} n_{2}}(t)-\varepsilon(t) \sum_{n_{1}^{\prime} n_{2}^{\prime}} z_{n_{1} n_{2}, n_{1}^{\prime} n_{2}^{\prime}} C_{n_{1}^{\prime} n_{2}^{\prime}}(t)
$$

where $E_{n_{1} n_{2}}=-1 /\left(2 n^{2}\right)$ is the unperturbed energy of the quantum state $\left(n_{1}, n_{2}\right)$ and $n$ is the principal quantum number given by $n=n_{1}+n_{2}+1$. The dipole coupling

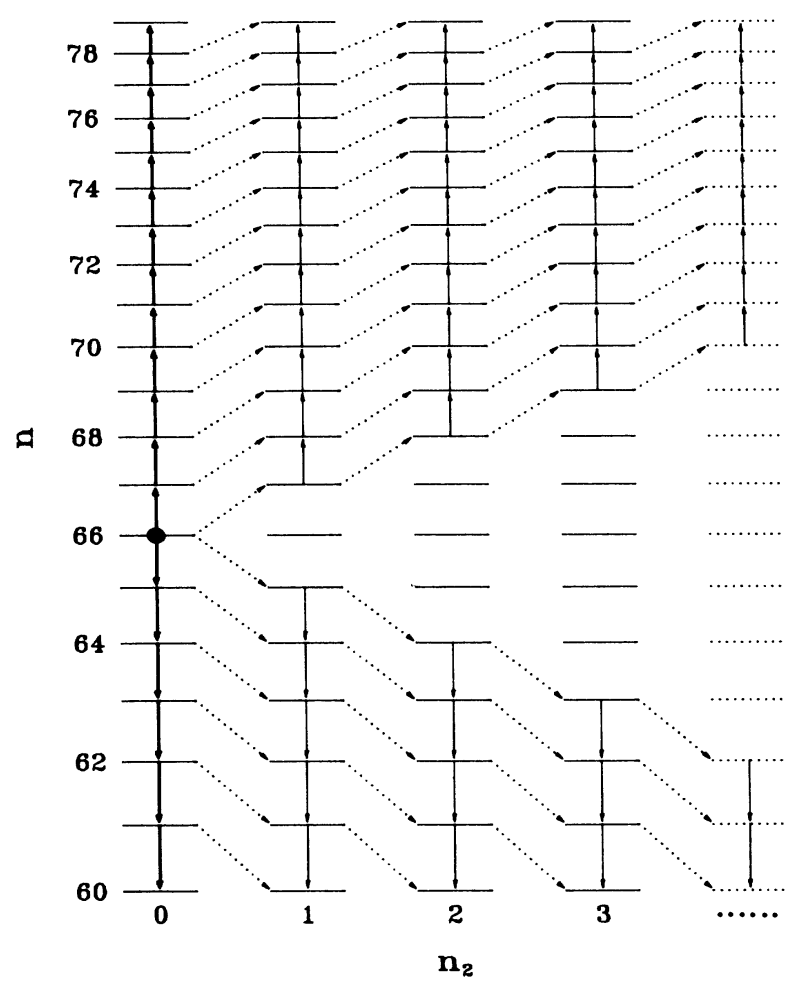

FIG. 1. Energy-level diagram and short-time population flow in the Rydberg $\mathbf{H}$ atom. The initial state (heavy dot) is located at $n=66, n_{2}=0$, and $m=0$. Only the $n_{2}=0$ ladder is considered in conventional $1 \mathrm{D}$ models. In reality, population can leak into $n_{2} \neq 0$ channels and excitation and ionization along $n_{2} \neq 0$ ladders can be subsequently developed. The complete solution of the problem thus involves the study of $2 \mathrm{D}$ dynamical evolution. 
matrix elements $z_{n_{1} n_{2}, n_{1}^{\prime} n_{2}^{\prime}}$ were computed using the formula by Gordon. ${ }^{13}$ The coupled equations (3) were solved by the Adam-Bashforth-Moulton predictorcorrector formulas of orders up to 12 to achieve high accuracy. The atom is assumed to be initially at the elongated quantum state $n_{0}=66$ or $n_{1}=65, n_{2}=m=0$ throughout this study. Since the Hamiltonian is periodic in time, namely, $\hat{H}(t+T)=\hat{H}(t)$, with $T=2 \pi / \omega$ being the period, the Floquet theorem ${ }^{10}$ can be invoked to facilitate the solution of long-time properties. Thus Eq. (1) can be rewritten as

$$
i \partial_{t} \hat{U}\left(t, t_{0}\right)=\hat{H}(t) \hat{U}\left(t, t_{0}\right),
$$

where $\hat{U}\left(t, t_{0}\right)$ is the time-evolution operator defined as

$$
\psi(t)=\hat{U}\left(t, t_{0}\right) \psi\left(t_{0}\right) \text { with } \hat{U}\left(t_{0}, t_{0}\right)=1 .
$$

The Floquet theorem asserts that

$$
\widehat{U}\left(t, t_{0}\right)=\widehat{P}\left(t, t_{0}\right) \exp \left[-i \widehat{Q}\left(t-t_{0}\right)\right],
$$

where $\hat{Q}$ is the time-independent quasienergy operator, and $\widehat{\boldsymbol{P}}\left(t, t_{0}\right)=\widehat{\boldsymbol{P}}\left(t+T, t_{0}\right)$ is periodic. Since $\hat{U}\left(t_{0}+n T, t_{0}\right)=\left[\hat{U}\left(t_{0}+T, t_{0}\right)\right]^{n}$, one sees that the timeevolution operator over one period, $\hat{U}\left(t_{0}+T, t_{0}\right)$, provides essentially all the information we ever need about the long-time behavior of the system. Further, in the truncated basis, $\hat{U}\left(t_{0}+T, t_{0}\right)$ may be diagonalized by some unitary transformation $S$,

$$
S^{\dagger} \hat{U}\left(t_{0}+T, t_{0}\right) S=\exp (-i D),
$$

where $D$ is a diagonal matrix. Thus

$$
\hat{U}\left(t_{0}+T, t_{0}\right)=S e^{-i D} S^{\dagger},
$$

whereupon

$$
\hat{U}\left(t_{0}+n T, t_{0}\right)=S e^{-i n D} S^{\dagger},
$$

The second way to solve Eq. (1) and take advantage of the Floquet theorem is via the so-called time-independent Floquet Hamiltonian method. ${ }^{10,14}$ In this approach, one transforms the periodically time-dependent Hamiltonian $\hat{H}(t)$ into an equivalent time-independent infinitedimensional Floquet matrix eigenvalue problem. However, as the number of quantum states and the number of photons involved are large, the dimensionality of the (truncated) Floquet matrix will be ultralarge. In Sec. IV, we shall discuss a method, the most probable path approach (MPPA), first introduced by Tietz and $\mathrm{Chu}^{15}$ in the study of multiphoton excitation of $\mathrm{SO}_{2}$. The method extends the artificial intelligence (AI) algorithms ${ }^{16}$ to search for the most important Floquet states, allowing the reduction of the dimensionality of the Floquet matrix into a manageable size and yet maintaining the essential dynamical information.

\section{EXCITATION DYNAMICS IN MICROWAVE-DRIVEN RYDBERG HYDROGEN ATOMS}

Two significant external parameters which determine the dynamical evolution of the system are the microwave frequency and field strength. Let us first establish the frequency-dependent excitation phenomena.

\section{A. Frequency-dependent excitation spectrum and dynamics}

A convenient measure of the extent of excitation along the energy space provided by the expectation value $\langle n(t)\rangle$ defined as

$$
\langle n(t)\rangle=\sum_{n^{\prime}} P\left(n_{0} \rightarrow n^{\prime} ; t\right) n^{\prime},
$$

where $P\left(n_{0} \rightarrow n^{\prime} ; t\right)$ is the population of the quantum state with the principal quantum number $n^{\prime}$ at time $t$. In general, $\langle n(t)\rangle$ exhibits quasiperiodic oscillations in time. Figure 2 shows the first maximum of the expectation values, $\langle n\rangle_{\text {max }}$, as a function of the (rescaled) frequency $\omega_{0}\left(\equiv \omega n_{0}^{3}\right)$ at the (rescaled) field strength $\varepsilon_{0}\left(\equiv \varepsilon n_{0}^{4}\right)=0.025$. We found that the excitation mechanisms depend strongly upon external field frequency and can be grouped roughly into three different regions: (i) The regions labeled by $B$ and $C$ showing the largest excitation are similar to the underthreshold large photoelectric ionization peaks recently reported by Casati et al. ${ }^{9}$ This region is bounded on the left by the classical chaotic threshold $\omega_{c}$ and on the right by the quantum delocalization border $\omega_{d}$. If the frequency is inside this window $\left(\omega_{c}<\omega_{0}<\omega_{d}\right)$, the major ionization mechanism is due to quantum diffusion as we shall show below. (ii) For frequency $\omega_{0}>\omega_{d}$, Casati et al. ${ }^{9}$ found that quantum localization occurs and the ionization probability drops sharply below the classical prediction. However, we observed in this region an infinite series of resonance peaks (labeled by $D, E, F$, etc.) with frequencies corresponding, respectively, to about one-photon resonance between $n=66 \rightarrow 69,66 \rightarrow 70$, and $66 \rightarrow 71$ etc. As will be shown below and in Sec. IV, near these frequencies, the dominant excitation (ionization) mechanism is due to multiphoton resonance processes. It is important to note that around peaks $B$ and $C$, no such initial resonance condi-

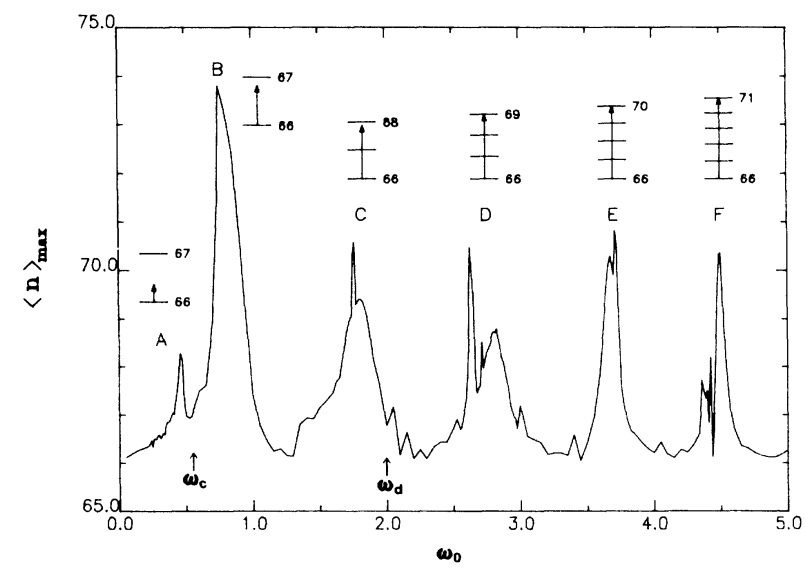

FIG. 2. Frequency-dependent excitation spectrum (see text for notations). Also shown here are schematic diagrams of the relative size of the field frequency $\omega$ and the energy spacings (not to scale). The physical parameters used are $\varepsilon_{0}=0.025$ and $n_{0}=66$ with $\varepsilon(t)=\varepsilon \cos \omega t$. 
tions are required as the dominant excitation mechanism is due to quantum diffusion. (iii) For frequency $\omega_{0}<\omega_{c}<<1$ (region $\mathbf{A}$ ), excitation is more difficult as it requires the absorption of several photons to reach even the nearby quantum states. As a result, higher microwave intensities are needed to achieve ionization. In this region, transitions mainly proceed through highorder processes, and a theoretical study of the excitation dynamics is currently in progress in our laboratory. In the current study, we shall focus mainly on the excitation mechanisms for $\omega_{0}>\omega_{c}$.

\section{B. Quantum diffusion versus multiphoton excitation}

First, let us investigate the physical origin of the unexpected high excitation in region $B$. Figure 3 shows the quasienergies (between -25.5 and $-24.5 \mathrm{~cm}^{-1}$ ) for frequencies nearby $\omega_{0}=0.8$. [For simplicity, only quasienergies associated with the $n_{2}=0$ ladder are displayed. Note that the unperturbed initial state is located at $E(n=66)=-25.2 \mathrm{~cm}^{-1}$. The quasienergy correlated with the initial state is indicated by $n=66, N=0$ in the figure where the second number $(N)$ is the Fourier (photon) index.] Notice the complicated avoided-crossing pattern just within $1 \mathrm{~cm}^{-1}$. In fact, all the quasienergy levels in this region are strongly perturbed and mixed and lose their identities. Once the system is in this frequency region, excitation is rather easy as the neighboring states interact almost evenly and there are many sets of $|\Delta n|=1$ and $|\Delta N|=1$ excitation pathways. This diffusivelike pattern ("quantum diffusion") is characterized by a series of successive single-photon absorption (or emission) steps between adjacent states.

In contrast, the corresponding quasi-energy-level avoided-crossing pattern for the $(D, E, F)$ resonant peak regions is relatively sparse. Figure 4 shows the quasienergy diagram for region $E$ for the field strength $\varepsilon_{0}=0.025$. Most quasienergy levels here maintain their identities and

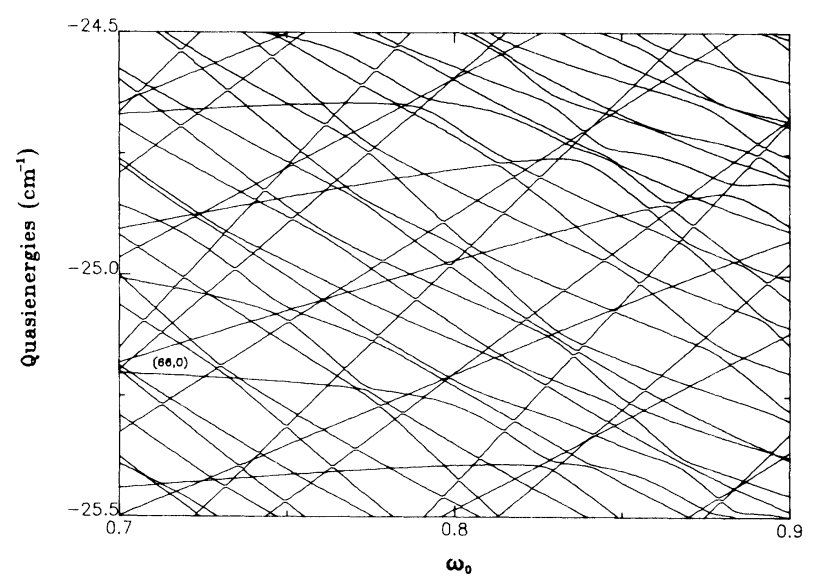

FIG. 3. Quasienergy avoided-crossing pattern near region $B$ $\left(\omega_{0} \sim 0.8\right)$ where quantum diffusion is the dominant excitation mechanism. Physical parameters same as Fig. 2. Only a small cross section (between -25.5 and $-24.5 \mathrm{~cm}^{-1}$ ) of quasienergies is shown. Most quasienergy levels are strongly perturbed and mixed and lose their identities. Excitaton is very efficient in this region.

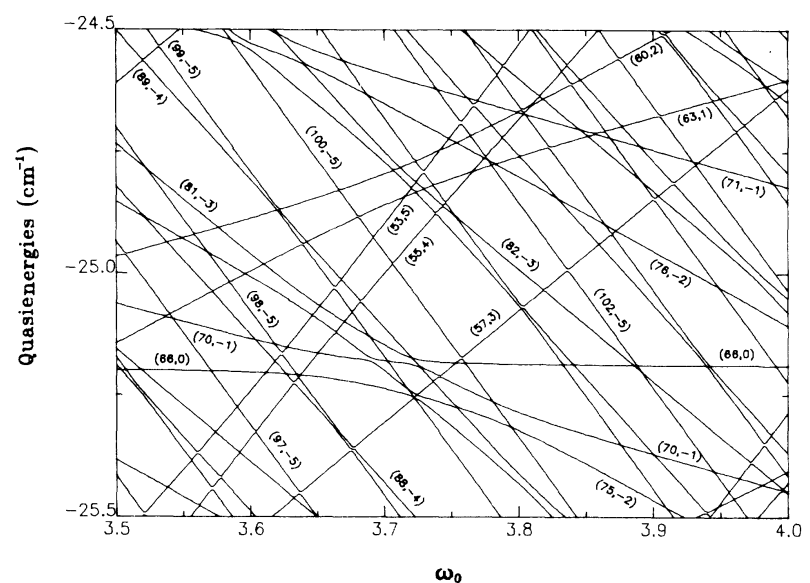

FIG. 4. Same as Fig. 3 except for $\omega_{0}$ near region $E\left(\omega_{0} \sim 3.66\right)$, where multiphoton resonant transitions are significant. Quasi-energy-level avoided crossings are more isolated in this region, and the identities of quasienergy levels can be easily distinguished as shown.

can be easily distinguished as indicated. The dominant excitation mechanism here is due to multiphoton resonant transitions (i.e., $\left|E_{n}-E_{n^{\prime}}\right| \cong N \hbar \omega$ with $\Delta n>1$ ) through the pairwise and isolated quasienergy anticrossing points. Since the dipole coupling strengths between adjacent states $(\Delta n= \pm 1)$ are always larger than those between $\Delta n>1$ states, the ionization probabilities in regions $D, E$, or $F$ will be correspondingly smaller than those in regions $A$ or $B$.

\section{Excitation and ionization pathways: 1D versus 2D}

We now focus our discussion on the most characteristic peak region labeled $B\left(\omega_{0}=0.8\right)$. The $2 \mathrm{D}$ calculations were performed by including quantum states in the range $50 \leq n \leq 250$ for each $n_{2}$ ladder $\left(n_{2}=0,1,2, \ldots\right.$; $\left.n_{1}=n-n_{2}-1\right)$. We found that three $n_{2}$ ladders $\left(n_{2}=0,1,2\right)$ are sufficient to achieve converged results for the case $\varepsilon_{0}=0.025$ and $\omega_{0}=0.8$ considered here. Figure 5 shows the time-dependent transition probabilities $P\left(n_{0}=66 \rightarrow n ; t\right)$ as a function of the state quantum number $n$ for (a) short-time regime $t=0.5 \tau$ (where $\tau$ is the period of microwave field $=2 \pi / \omega \cong 55 \mathrm{ps}$ ), (b) and (c) intermediate time regimes $t=5 \tau$ and $10 \tau$ and (d) the longtime regime $t=100 \tau$. As we see here, the $1 \mathrm{D}$ model mimics very well the $2 \mathrm{D}$ results at short times $(t<1 \tau)$, indicating the dynamical evolution is mainly driving along the $n_{2}=0$ ladder. At longer times, while the major flux is still "localized" in certain ranges near $n_{0}$ and well described by the 1D model, the evolution of the higher quantum states (which is mainly responsible for ionization) shows a large departure from the 1D results. Note that the localization phenomenon mainly occurs in the $n_{2}=0$ ladder and prevails in the large times. The evolution in $n_{2} \neq 0$ ladders is more delocalized and shows qualitatively different behavior from the dynamics in the $n_{2}=0$ ladder. In Fig. 5(d), only the total 2D transition probabilities are shown along with the $1 \mathrm{D}$ result. In this longer-time regime $(t=100 \tau)$, the $n$-dependent popula- 
tions (of higher quantum states) in each $n_{2}$ ladder are roughly within the same order of magnitude and difficult to distinguish in the logarithm graphic scale. This also indicates that more $n_{2}$ ladders will be required to achieve convergence in the dynamical calculations for $t \gg 100 \tau$.
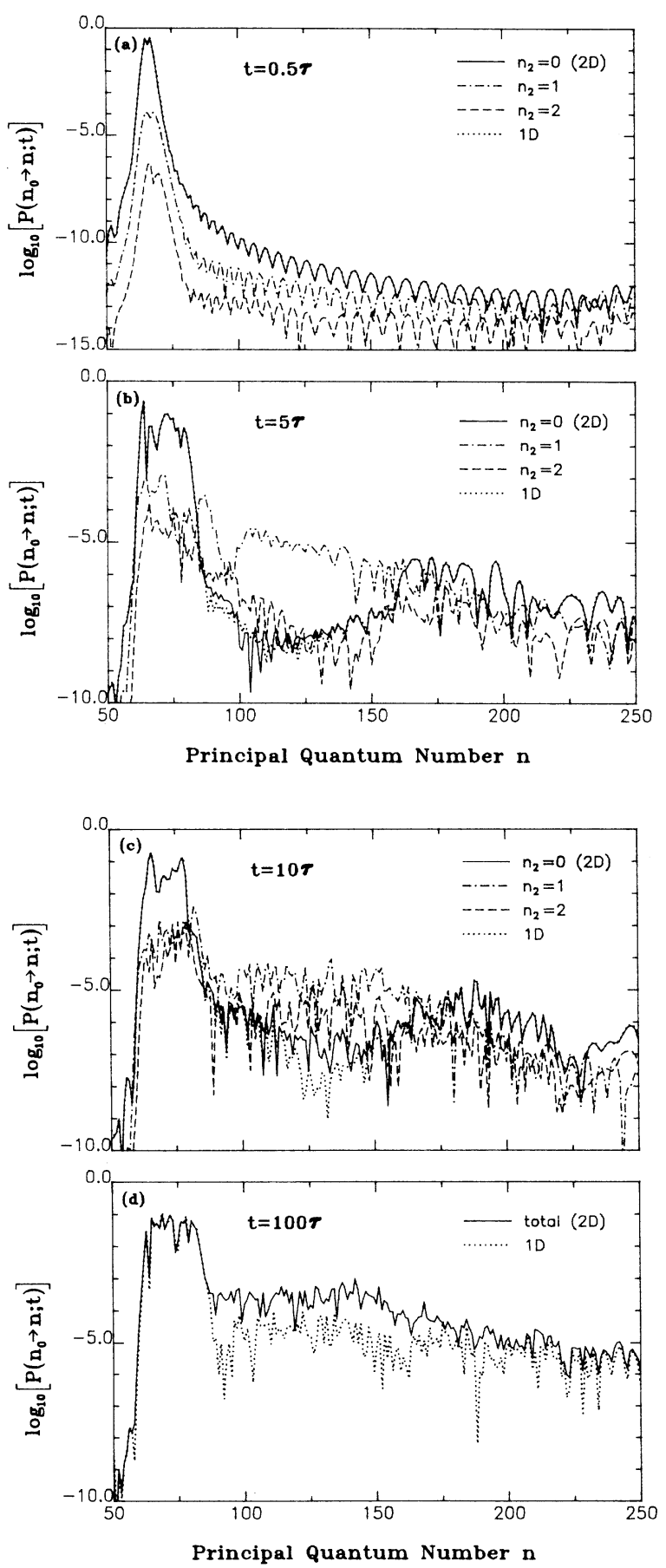

FIG. 5. Time-dependent 2D transition probabilities $P\left(n_{0}=66 \rightarrow n ; t\right)$ as a function of state quantum number $n$ at $\omega_{0}=0.8$ and $\varepsilon_{0}=0.025$. (a) $t=0.5 \tau$, (b) $t=5 \tau$, (c) $t=10 \tau$, and (d) $t=100 \tau$, where $\tau$ is the period of the microwave field.
Figure 6 shows the comparison of the total flux flowing into the $n \geq 100$ states, a measure of the ionization probabilities, for (a) $\varepsilon(t)=\varepsilon \sin \omega t$ and (b) $\varepsilon(t)=\varepsilon \cos \omega t$. The $1 \mathrm{D}$ model (dotted line) is seen to underestimate significantly the ionization probabilities in both cases. The most notable result is the dominance of the diffusion flux developed in the $n_{2}=1$ and 2 ladders in the time span shown. For even longer-time development $(t>100 \tau)$, evolution in higher $n_{2}$ ladders $\left(n_{2}>2\right)$ will be increasingly important. This shows clearly that ionization takes place not along the $n_{2}=0$ ladder, but through the $n_{2} \neq 0$ ladders. Figures $6(\mathrm{a})$ and $6(\mathrm{~b})$ also reveal that the way of turning on the microwave field does not change qualitatively the subsequent dynamical evolution. To show the subtlety of the effect of turning on the field, the short to intermediate temporal ( $t=0$ to $10 \tau)$ behaviors of Fig. 6 are enlarged and displaced in Fig. 7. The solid lines are the results for $\varepsilon(t)=\varepsilon \sin \omega t$, while the dotted lines are for $\varepsilon(t)=\varepsilon \cos \omega t$. Both results reveal a time delay of about five field oscillations before the ionization flux becomes appreciable. Notice the interesting stepwise behavior - the ionization probability raises up suddenly
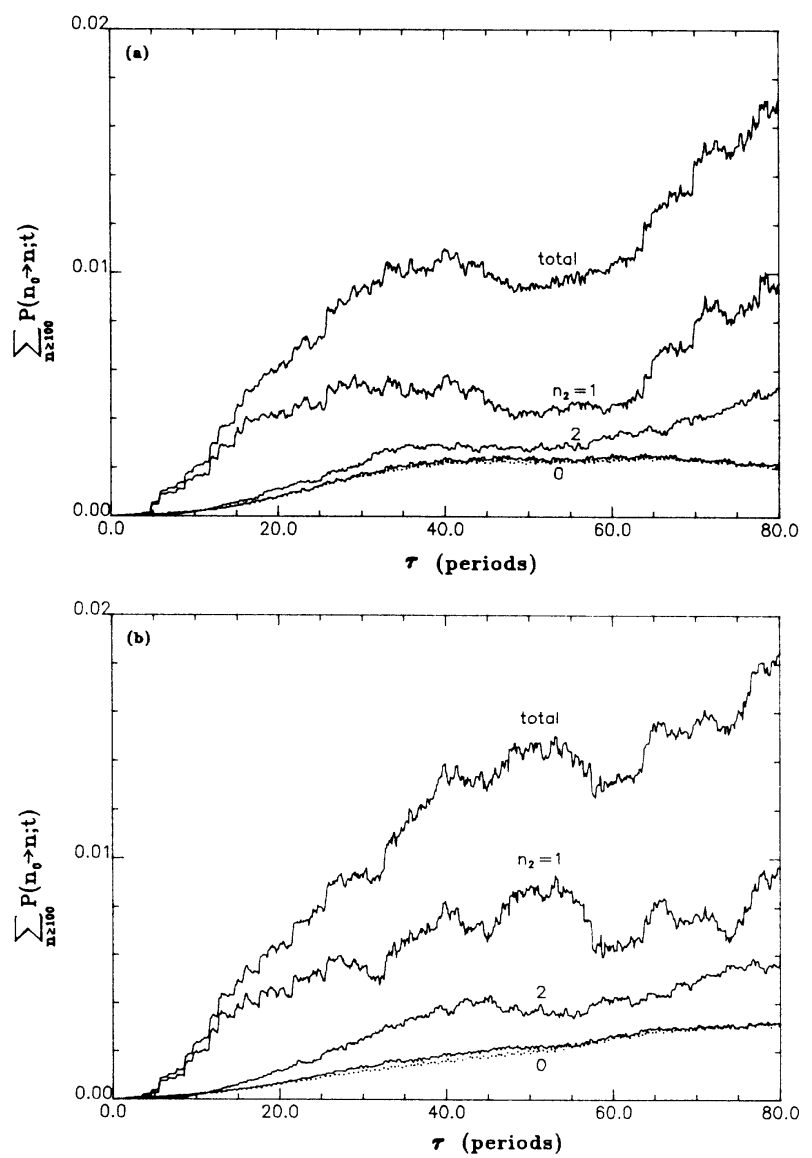

FIG. 6. Time-dependent $2 \mathrm{D}$ ionization probabilities at $\omega_{0}=0.8, \varepsilon_{0}=0.025$, and $n_{0}=66$ for (a) $\varepsilon(t)=\varepsilon \sin \omega t$ and (b) $\varepsilon(t)=\varepsilon \cos \omega t$. The 1D results (shown by the dotted curves) are seen to underestimate the ionization probabilities significantly. 


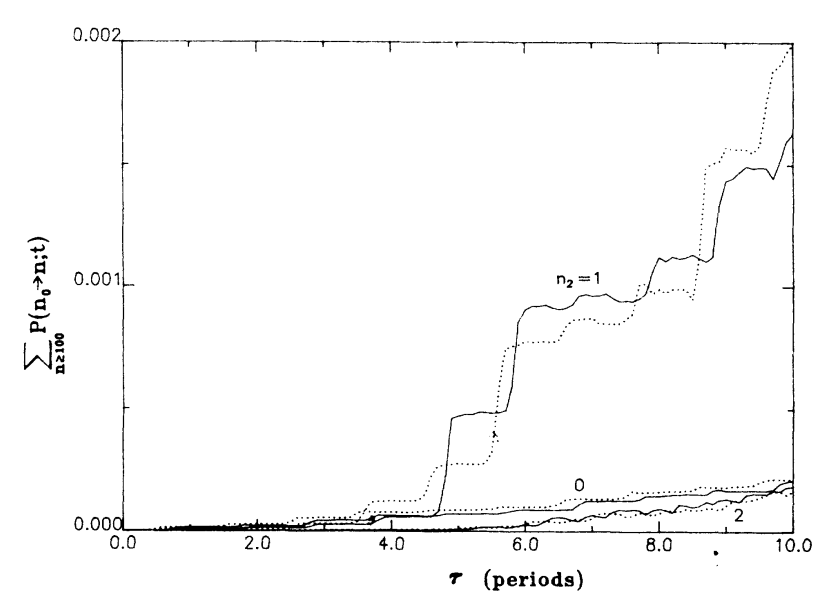

FIG. 7. Same as Fig. 6 except the short to intermediate temporal ionization probabilities are enhanced to show the subtlety of the effect of field turn-on. Solid curves are for $\varepsilon(t)=\varepsilon \sin \omega t$ and dotted curves are for $\varepsilon(t)=\varepsilon \cos \omega t$.

and then stays in a plateau for about one period before the next jump. The behavior with the $\varepsilon \sin \omega t$ microwave field shows a $\tau / 4$ time delay from that of the $\varepsilon \cos \omega t$ field.

We note that the field strength used in this calculation $\left(\varepsilon_{0}=0.025\right)$ is larger than the classical critical value $\varepsilon_{c}=0.02 \omega_{0}^{-1 / 3}$ but smaller than the threshold for quantum delocalization ${ }^{9} \varepsilon_{q}=\omega_{0}^{7 / 6} / \sqrt{6 n_{0}}$. (These threshold values are only approximate and are derived from 1D models for the $\omega_{0} \geq 1$ case. They are used here merely for comparison purposes.) According to classical-dynamical studies, the motion of the hydrogenic electron will exhibit chaotic behavior and follow a diffusive ionization law. The results shown in Fig. 5 demonstrate a behavior corresponding to the so-called quantum localization in classically chaotic systems which was known also in other model problems ${ }^{4,17}$ as well as in the $1 \mathrm{D}$ atomic $H$ model. $^{7}$ Our 2D results, however, reveal for the first time, the detailed dynamical subtleties in Rydberg-atom excitation. The results shown in Fig. 5 indicate the existence of the quantum localization phenomenon even for $\omega_{0}<1$. This is consistent with the recent experimental result of Bayfield and Sokol ${ }^{18}$ where they found similar phenomena for the parameters $\omega_{0}=0.880$ and $\varepsilon_{0}=0.021$ (which are close to our values) and $n_{0}=72$.

\section{Intensity-dependent excitation and ionization}

Also significant to study is the effect of field strength on the excitation and ionization mechanisms. First we have considered weaker field strengths $\left(\varepsilon_{0}<0.025\right)$ at $\omega_{0}=0.8$. We found that similar behavior, such as those shown in Figs. 5 and 6, prevails. As a general observation, the quantum localization in classically chaotic regions can take place as long as $\varepsilon_{c}<\varepsilon_{0}<\varepsilon_{q}$. The quantum localization here is characterized by the dominance of the population around the initial state $n_{0}$ stretching along the $n_{2}=0$ ladder with the localization length depending upon the field strength. At the same time, however, quantum diffusion in higher quantum states can occur in all the $n_{2}$ ladders. In fact, it is the time development of the higher quantum levels which is mainly responsible for ionization. The dominant ionization channel is seen not via the $n_{2}=0$ ladder (as often assumed in the $1 \mathrm{D}$ model) but through the diffusive excitation in the $n_{2} \neq 0$ ladders.

Let us now consider the higher-field-strength regime. In general, we found as the field strength increases, the quantum localization border along the $n_{2}=0$ ladder stretches to higher quantum states more rapidly, and the 1D model improves. In fact, at sufficiently high field strengths, such that $\varepsilon_{0}>\varepsilon_{q}$, quantum delocalization can indeed appear. Figures $8(a)$ and $8(\mathrm{~b})$ show, respectively, the 2D ionization probabilities for $\varepsilon(t)=\varepsilon \sin \omega t$ and $\varepsilon(t)=\varepsilon \cos \omega t$, both at the field strength $\varepsilon_{0}=0.05$ (which is now larger than $\varepsilon_{q}$ ). The microwave power is strong enough to drive the electron to very highly excited states or continuums through the $n_{2}=0$ ladder before a sufficient amount of diffusion to the $n_{2} \neq 0$ ladders occurs. Figure 8 shows that the 1D ionization flux (dotted line) agrees with the total $2 \mathrm{D}$ ionization flux reasonably well even up to sufficiently long times. The way of turning on the field $(\sin \omega t$ or $\cos \omega t)$ again does not appear to cause a significant effect on the time development of the ionization flow. Based on the above study, we can conclude
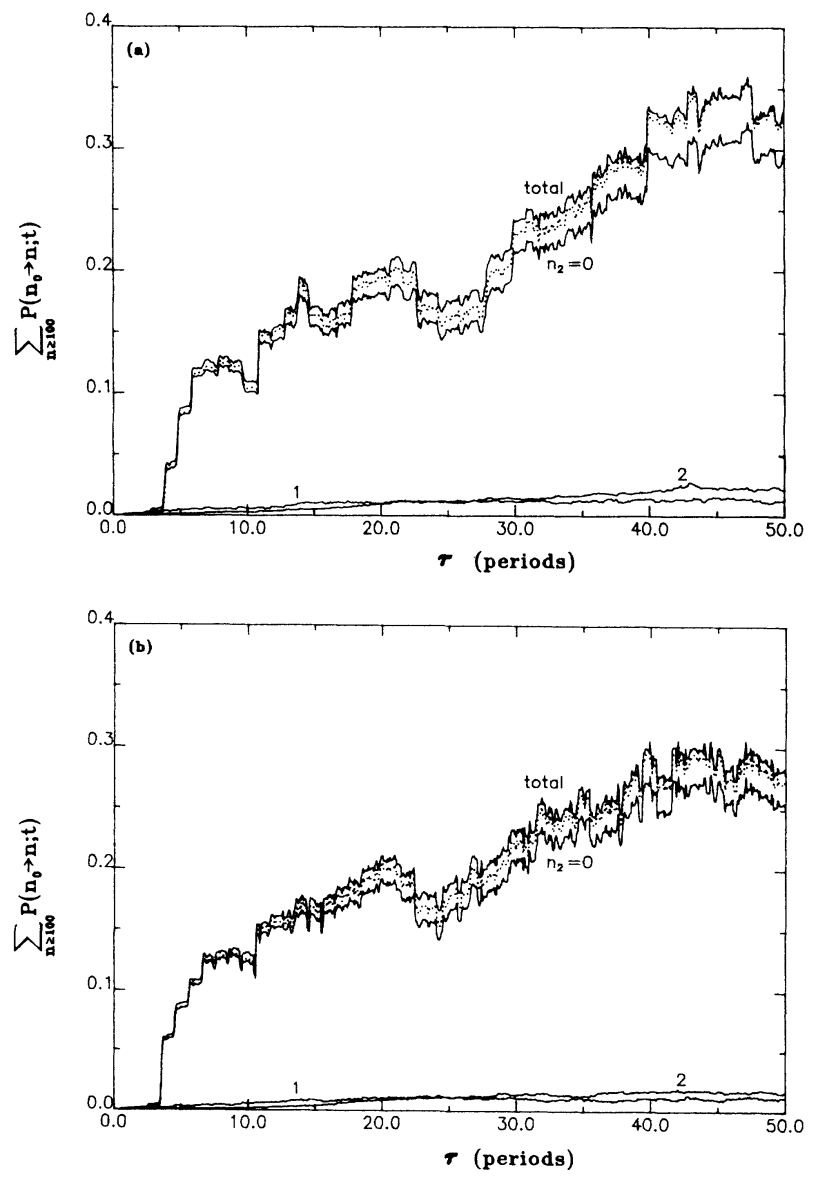

FIG. 8. Same as Fig. 6 except the field strength has been increased to $\varepsilon_{0}=0.05$, which is larger than the quantum delocalization threshold $\varepsilon_{q}$. The $1 \mathrm{D}$ results agree well with the total 2D results for both (a) $\varepsilon(t)=\varepsilon \sin \omega t$ and (b) $\varepsilon(t)=\varepsilon \cos \omega t$. 
that the 1D model seems to be capable of providing an adequate description of the quantal diffusive ionization, provided that $\varepsilon_{0}>\varepsilon_{q}$ and the evolution time is not too large.

\section{E. Classical versus "quantum" chaos}

Now we turn to the study of another interesting aspect of the problem, namely, the stability of quantum diffusive motion in the classically chaotic regime. Again we use the example with $\varepsilon_{0}=0.05$ and $\omega_{0}=0.8$. Only the $1 \mathrm{D}$ results are needed to illustrate the essential dynamics at this field strength. The quantal expectation values of the coordinate $\langle x(t)\rangle$ and the momentum $\langle p(t)\rangle$ of the electron are shown as a function of the evolution time in Fig. 9. It is seen that both $\langle x(t)\rangle$ and $\langle p(t)\rangle$ oscillate in time with the former gradually increasing and the latter gradually decreasing in magnitude. Particularly intriguing is the quantal phase-space diagram $[\langle p(t)\rangle$ versus $\langle x(t)\rangle]$ shown in Fig. 10. It indicates on average that the electron exhibits stable quasiperiodic motion around the nucleus, albeit its distance from the nucleus gradually increases toward ionization. On the other hand, a nonlinear classical-dynamical calculation would show much stronger instability and wandering motion in the phase space in this classically chaotic regime.

Additional information on the stability as well as the more deterministic nature of the quantum diffusive motion can be seen in Fig. 11. Here the modulus of the autocorrelation function $C(\tau)$,

$$
C(\tau)=\langle\psi(0) \mid \psi(\tau)\rangle,
$$

as well as its Fourier transform are displayed. We see that $|C(\tau)|$ shows quasiperiodic oscillations in time and its Fourier transform exhibits recurrence peaks. Thus the population delocalization and quantum diffusion phenomena reported above are not truly dynamical chaos. [The "quantum" chaos would be characterized by a fast decay of the correlation function $C(\tau)$ to zero. ${ }^{11,19}$ This is not seen in Fig. 11(a).] The quantum limitation of clas-

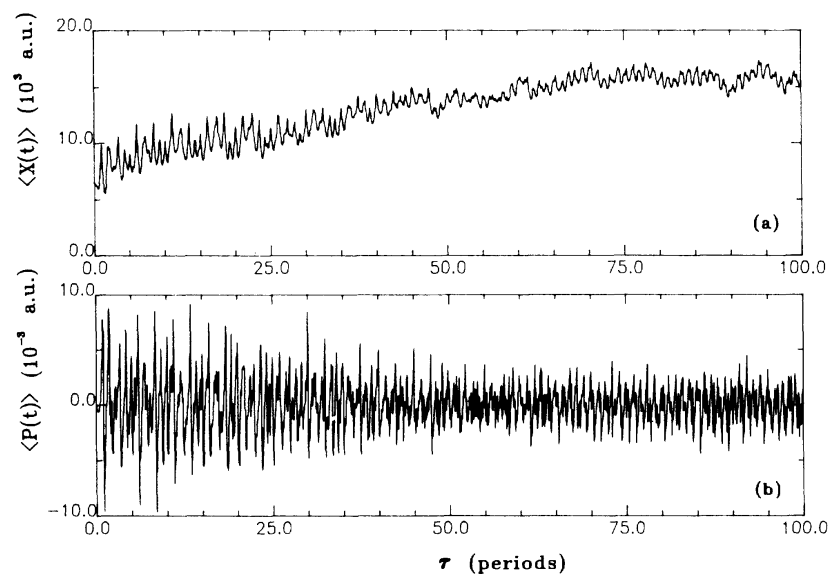

FIG. 9. Expectation values of $x(t)$ and $p(t)$ as a function of time (in units of period $\tau$ ) at $\omega_{0}=0.8, \varepsilon_{0}=0.05$, and $n_{0}=66$ for $\varepsilon(t)=\varepsilon \cos \omega t$.

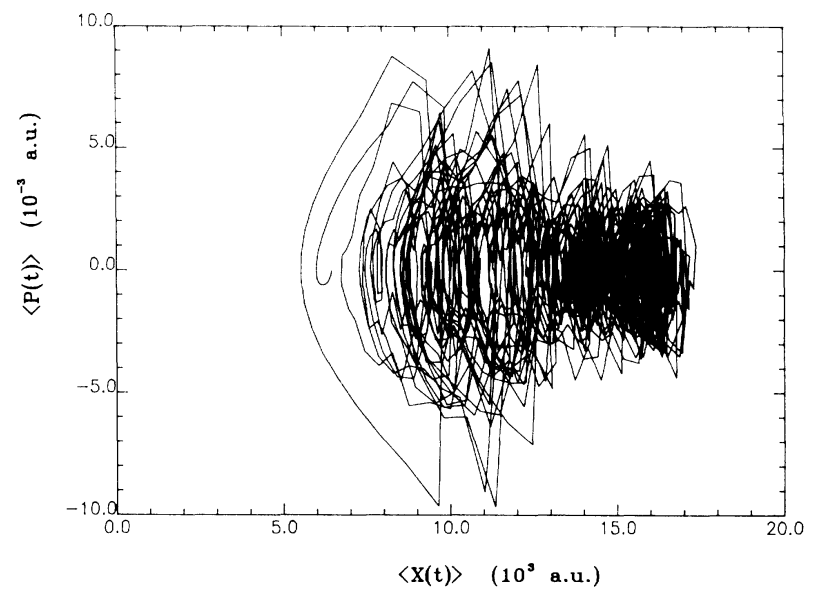

FIG. 10. "Quantal" phase-space diagram $\langle P(t)\rangle$ vs $\langle X(t)\rangle$. Physical parameters are the same as in Fig. 9. This diagram suggests that quantum diffusive motion can be stable even if the corresponding classical system is chaotic.

sical chaos is clearly demonstrated in our results. The effect of continuum (which is not included in this study) deserves further consideration. However, a recent study of the dynamics of periodically perturbed surface-state electrons shows that the effect of continuum on the quantum-mechanical dynamics in the classically chaotic region is not significant. ${ }^{20}$

\section{DYNAMICS OF MULTIPHOTON RESONANT EXCITATION: MOST PROBABLE PATH APPROACH-ARTIFICIAL INTELLIGENCE ALGORITHMS}

The excitation-ionization mechanisms for regions labeled by $D, E$, and $F$ in Fig. 2 are quite different from those of regions $B$ and $C$. Let us consider a specific example, namely, a frequency in peak $E$ (Fig. 2) corresponding to $\omega_{0}=3.66$. At this frequency, it takes nine photons to reach $n \cong 1500$ (from $n_{0}=66$ ) and the tenth photon to

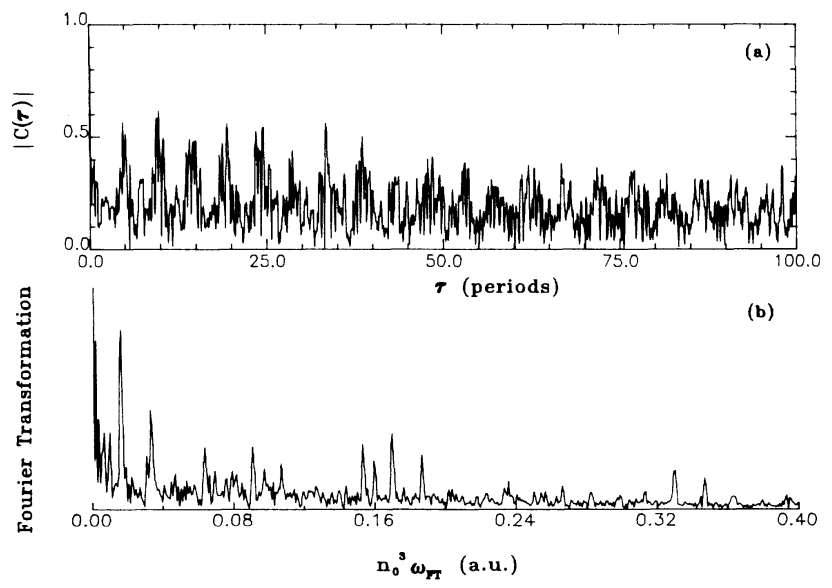

FIG. 11. Autocorrelation function and its Fourier transform. Physical parameters are the same as in Fig. 9. 
ionize the atom. Diffusion processes still take place, but it is the multiphoton resonance transitions $\left(\left|E_{i}-E_{j}\right|\right.$ $\cong N \hbar \omega$ where $|i\rangle$ and $|j\rangle$ are not adjacent levels) which are mainly responsible for the ionization. As the number of quantum states involved is huge, it is not feasible to numerically solve the coupled equations in Eq. (3). An alternative approach is to adopt the time-independent Floquet matrix method. ${ }^{10,14}$ However, the dimensionality of the Floquet matrix can easily become ultralarge, depending on how many quantum states and Floquet photon blocks are included. To circumvent this large dimensionality problem, we use a modified version of the socalled most probable path approach introduced previously in the study of the multiphoton excitation and dissociation of triatomic molecules. ${ }^{15}$ The method recognizes the fact that while the "exact" Floquet matrix is infinitely large, the majority of the atom-field Floquet states is unimportant due to either extremely large detunings or very small coupling matrix elements. The MPPA uses $N$ th-order perturbative terms of the type

$$
\begin{aligned}
& \left\langle\alpha_{N}|\mu| \alpha_{0}\right\rangle_{p} \\
& \quad=\prod_{k=0}^{N-1}\left\langle\alpha_{k+1}|\mu| \alpha_{k}\right\rangle / \prod_{k=2}^{N}\left(E_{k-1}-E_{0}-n_{k-1} \omega\right)
\end{aligned}
$$

to preselect which atom-field Floquet states are important at each step of the multiphoton process. Here, $\alpha_{i}$ is the atomic state $|i\rangle, E_{i}$ is the unperturbed energy of $\alpha_{i}$, $n_{i}$ is the number of photons associated with the atomfield state $\left|\alpha_{i} n_{i}\right\rangle$, and the subscript $p$ denotes a particular path through the intermediate states. Note that $n_{i+1}=n_{i} \pm 1$ due to dipole selection rules. The diagonal couplings (which have significant effects on ac Stark shifts of energy levels) are included in our calculation.

The procedure is derived from algorithms which utilize artificial intelligence to prune the number of choices at each node (photon order, in our case) of a decision tree. The MPPA begins by calculating all possible secondorder perturbative terms. The $N_{p}$ largest couplings (where $N_{p}$ is the number of paths to keep at each step) are chosen as the most probable paths through secondorder. The initial state (root node) and the intermediate states of the chosen paths are marked as important and are used in the final calculations. At each iterative step, the method calculates all possible $(N+1)$ st-order couplings (paths) using only the $N_{p} N$ th-order paths saved in the last iteration. The $(N+1)$ st-order couplings are then examined and the largest $N_{p}$ are saved for further traversal. $N$ th-order states which have now become intermediate to a large $(N+1)$ st-order path are "important" and are marked for later use. By iterating long enough, one can traverse the entire Floquet atom-field basis space, saving only those states which are important to the various $i$ th-order processes. Improved AI algorithms ${ }^{16}$ such as dynamic programming or $A^{*}$ can be used to facilitate the search of optimal paths. The reduction of the basis set is quite dramatic, leading to many orders of magnitude savings in computer time. For the case $\omega_{0}=3.66$, $\varepsilon_{0}=0.025$, for example, MPPA allows the reduction of the dimensionality of the Floquet matrix $\hat{H}_{F}$ from about $10^{5}$ to an effective matrix $\hat{H}_{\text {MPPA }}$ of about $10^{3}$ in dimension and still maintains reasonable accuracy. (Up to 1200 quantum states in each $n_{2}$ ladders are considered in the present study.) The study of multiphoton excitation (time-dependent or time-averaged) dynamics thus reduces to the solution of a time-independent eigenvalue problem. For example, the long-time averaged transition probability from the initial atomic state $|\alpha\rangle$ to a final atomic state $|\beta\rangle$ is given by ${ }^{10}$

$$
\bar{P}_{\alpha \rightarrow \beta}=\sum_{l} \sum_{\gamma^{\prime} l^{\prime}}\left|\left\langle\beta l \mid \lambda_{\gamma^{\prime} l^{\prime}}\right\rangle\left\langle\lambda_{\gamma^{\prime} l^{\prime}} \mid \alpha 0\right\rangle\right|^{2},
$$

where $\left|\lambda_{\gamma^{\prime} l^{\prime}}\right\rangle$ are the quasienergy eigenfunctions of $\hat{H}_{\text {MPPA }}$, and $\left\langle\beta l \mid \lambda_{\gamma^{\prime} l^{\prime}}\right\rangle$ is the projection of $\left|\lambda_{\gamma^{\prime} l^{\prime}}\right\rangle$ onto the unperturbed atom-field state $|\beta l\rangle$ with $\beta$ being the atomic index and $l$ the Fourier photon index.

The accuracy of MPPA results can be further improved by invoking an extension of the generalized Van Vleck (GVV) nearly degenerate perturbation technique. ${ }^{21,22}$ The general idea behind the GVV technique is to block-diagonalize the original Floquet matrix $\hat{H}_{F}$ so that the coupling between the model space (consisting of nearly degenerate and strongly coupled Floquet states of interest) and the remainder of the configuration space (called the external space) diminishes to a desired order. One important feature of the GVV approach is that if the perturbed-model-space wave functions are exact to the $n$th order, the corresponding quasienergy eigenvalues in the model space will be accurate to the $(2 n+1)$ st order. In our current problem, we can treat $\hat{H}_{\mathrm{MPPA}}$ as the unperturbed-model-space Hamiltonian. Higher-order corrections to $\hat{H}_{\text {MPPA }}$ can be achieved by systematically taking into account, order by order, the couplings of the model space with the Floquet states in the external space (i.e., those not chosen by the MPPA procedure). This gives rise to a new effective Hamiltonian $\widehat{H}_{\text {MPPA-GVV }}$. For the Rydberg $\mathrm{H}$-atom problem, we found that the second-order GVV correction is sufficient to provide accurate dynamical information about multiphoton reso-

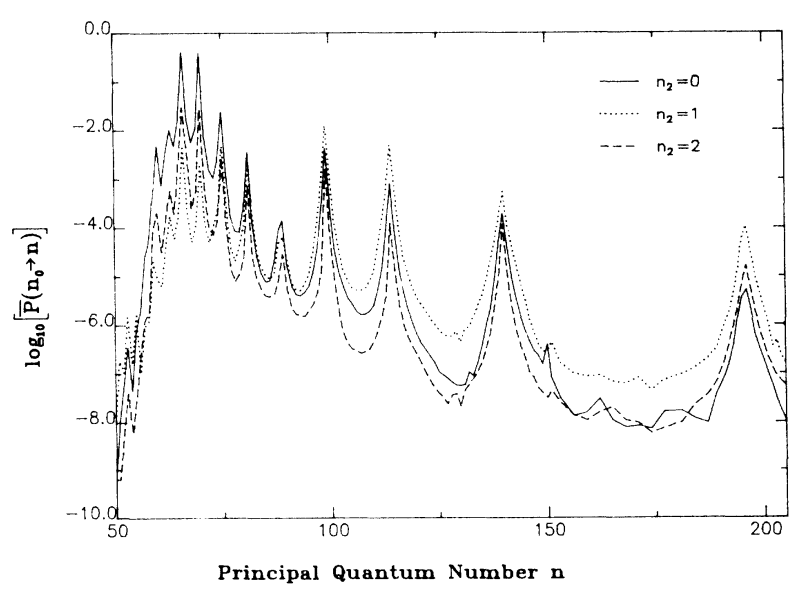

FIG. 12. Long-time average transition probabilities $\bar{P}\left(n_{0}=66 \rightarrow n\right)$ vs $n$ at $\omega_{0}=3.66$ and $\varepsilon_{0}=0.025$ for $\varepsilon(t)=\varepsilon \cos \omega t$. The multiphoton resonance structure is clearly seen. These results are obtained from the MPPA-GVV method. 
nant excitation.

Figure 12 shows the 2D MPPA-GVV results for the long-time averaged populations $\bar{P}\left(n_{0}=66 \rightarrow n\right)$ as a function of the state quantum number $n$ at $\omega_{0}=3.66$ and $\varepsilon_{0}=0.025$. Three $n_{2}$ ladders (i.e., $n_{2}=0,1$, and 2) are included in the $2 \mathrm{D}$ calculations. The multiphoton resonance structure is clearly seen. It is interesting to note that, similar to the case of $\omega_{0}=0.8$, the population is concentrated along the $n_{2}=0$ ladder for $n<100$, while the $n_{2}>0$ ladders are the dominant channels for the time development of higher Rydberg states and ionization.
When the intensity is increased to $\varepsilon_{0}=0.05$, we again find (not shown here) that the population is primarily driven along the $n_{2}=0$ ladder for all quantum numbers, and that resonance peaks are substantially shifted, broadened, and enhanced.

\section{ACKNOWLEDGMENTS}

This work was supported in part by the U.S. Department of Energy (Division of Chemical Sciences), by the John Simon Guggenheim Foundation (S.-I.C.), and by the University of Kansas-General Research Fund.
${ }^{1}$ J. E. Bayfield and L. A. Pinnaduwage, Phys. Rev. Lett. 54, 313 (1985); K. A. H. Van Leeuwen, G. V. Oppen, S. Renwick, J. B. Bowlin, P. M. Koch, R. V. Jensen, O. Rath, D. Richards, and J. G. Leopold, ibid. 55, 2231 (1985).

${ }^{2}$ G. Casati, B. V. Chirikov, D. L. Shepelyansky, and I. Guarneri, Phys. Rep. 154, 77 (1987).

${ }^{3}$ G. Casati, B. V. Chirikov, I. Guarneri, and D. L. Shepelyansky, Phys. Rev. Lett. 59, 2927 (1987).

${ }^{4}$ R. V. Jensen, Phys. Rev. Lett. 49, 1365 (1982); Phys. Rev. A 30, 386 (1984).

${ }^{5}$ J. N. Bardsley, B. Sundaram, L. A. Pinnaduwage, and J. E. Bayfield, Phys. Rev. Lett. 56, 1007 (1986).

${ }^{6}$ N. B. Delone, V. P. Krainov, and D. L. Shepelyansky, Usp. Fiz. Nauk 140, 355 (1983) [Sov. Phys.-Usp. 26, 551 (1983)]

${ }^{7}$ G. Casati, B. V. Chirikov, and D. L. Shepelyansky, Phys. Rev. Lett. 53, 2525 (1984).

${ }^{8}$ G. Casati, B. V. Chirikov, I. Guarneri, and D. L. Shepelyansky, Phys. Rev. Lett. 56, 2437 (1986).

${ }^{9}$ G. Casati, B. V. Chirikov, D. L. Shepelyansky, and I. Guarneri, Phys. Rev. Lett. 57, 823 (1986).

${ }^{10}$ S. I. Chu, Adv. At. Mol. Phys. 21, 197 (1985), and references therein.

${ }^{11}$ S. I. Chu, Adv. Chem. Phys. 73, 739 (1988).

${ }^{12}$ Second International Laser Science Conference, Seattle, Washington, 1986 (unpublished).

${ }^{13}$ W. Gordon, Ann. Phys. 2, 1031 (1929).

${ }^{14}$ J. H. Shirley, Phys. Rev. 138, B979 (1965)

${ }^{15}$ J. V. Tietz and S. I. Chu, Chem. Phys. Lett. 101, 446 (1983).

${ }^{16}$ P. H. Winston, Artificial Intelligence (Addison-Wesley, Reading, MA, 1979).

${ }^{17}$ See, for example, S. Fishman, D. R. Grempel, and R. E. Prange, Phys. Rev. A 29, 1639 (1984).

18J. E. Bayfield and D. W. Sokol, Phys. Rev. Lett. 61, 2007 (1988).

${ }^{19}$ M. Shapiro and G. Goelman, Phys. Rev. Lett. 53, 1714 (1984).

${ }^{20}$ R. Blümel and U. Smilansky, Phys. Rev. 30, 1040 (1984).

${ }^{21}$ B. Kirtman, J. Chem. Phys. 49, 3890 (1968); 75, 798 (1981); P. R. Certain and J. O. Hirschfelder, J. Phys. Chem. 52, 5977 (1970); P. K. Aravind and J. O. Hirschfelder, ibid. 88, 4788 (1984).

${ }^{22}$ T. S. Ho and S. I. Chu, Phys. Rev. A 31, 659 (1985); 32, 377 (1985). 